Document downloaded from:

http://hdl.handle.net/10251/36183

This paper must be cited as:

Kazarin, SL.; Martínez Pastor, A.; Pérez-Ramos, MD. (2011). On the Sylow graph of a finite group. Israel Journal of Mathematics. 186(1):251-271. doi:10.1007/s11856-011-0138$\mathrm{x}$.

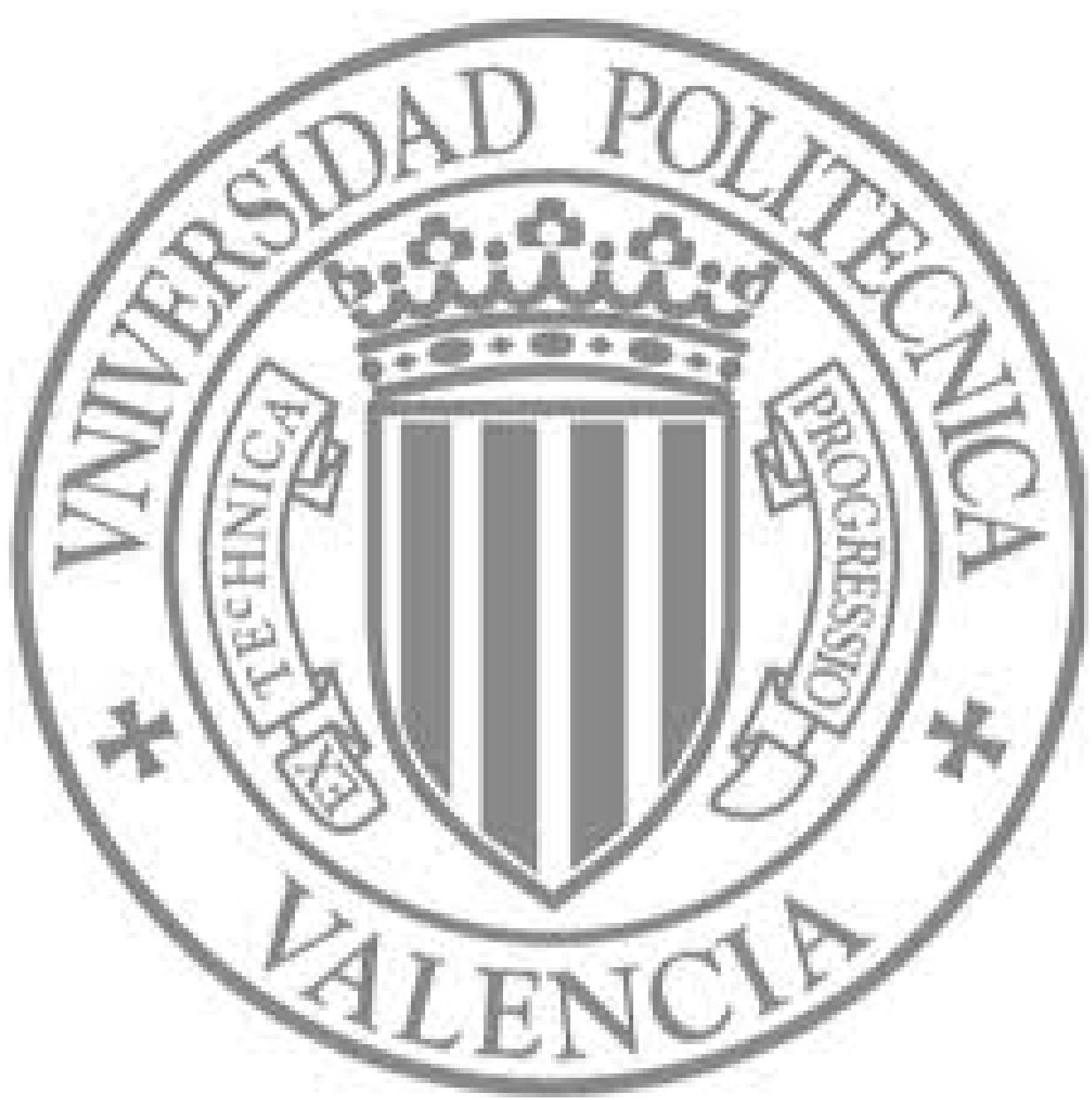

The final publication is available at

http://link.springer.com/article/10.1007\%2Fs11856-011-0138-x

Copyright Springer Verlag (Germany) 


\title{
On the Sylow graph of a group and Sylow normalizers*
}

\author{
L. S. Kazarin \\ Department of Mathematics, Yaroslavl P. Demidov State University \\ Sovetskaya Str 14, 150014 Yaroslavl, Russia \\ E-mail: Kazarin@uniyar.ac.ru \\ A. Martínez-Pastor \\ Escuela Técnica Superior de Ingeniería Informática, \\ Instituto Universitario de Matemática Pura y Aplicada IUMPA-UPV \\ Universidad Politécnica de valencia \\ Camino de Vera, s/n, 46022 Valencia, Spain \\ E-mail: anamarti@mat.upv.es
}

and

M. D. Pérez-Ramos

Departament d'Àlgebra, Universitat de València

C/ Doctor Moliner 50, 46100 Burjassot (València), Spain

E-mail: Dolores.Perez@uv.es

\begin{abstract}
Let $G$ be a finite group and $G_{p}$ be a Sylow $p$-subgroup of $G$ for a prime $p$ in $\pi(G)$, the set of all prime divisors of the order of $G$. The automiser $A_{p}(G)$ is defined to be the group $N_{G}\left(G_{p}\right) / G_{p} C_{G}\left(G_{p}\right)$. We define the Sylow graph $\Gamma_{A}(G)$ of the group $G$, with set of vertices $\pi(G)$, as follows: Two vertices $p, q \in \pi(G)$ form an edge of $\Gamma_{A}(G)$ if either $q \in \pi\left(A_{p}(G)\right)$ or $p \in \pi\left(A_{q}(G)\right)$. The following result is obtained:
\end{abstract}

*The second and third authors have been supported by Proyecto MTM2007-68010C03-03, Ministerio de Educación y Ciencia and FEDER, Spain.

The first author thanks the Universitat de València and the Universidad Politécnica de Valencia for their warm hospitality during the preparation of this paper. 
Theorem: Let $G$ be a finite almost simple group. Then the graph $\Gamma_{A}(G)$ is connected and has diameter at most 5 .

We also show how this result can be applied to derive information on the structure of a group from the normalizers of its Sylow subgroups.

Keywords: Finite groups, Simple groups, Sylow normalizers, Saturated formations.

MSC2000: 20E32, 20D20, 20F17

\section{Introduction}

All groups considered are finite.

Let $G$ be a finite group and $G_{p}$ be a Sylow $p$-subgroup of $G$ for any $p \in \pi(G)$, where $\pi(G)$ denotes the set of prime divisors of the order of $G$. Then the automiser $A_{p}(G)$ is defined to be the group $N_{G}\left(G_{p}\right) / G_{p} C_{G}\left(G_{p}\right)$. We construct the Sylow graph $\Gamma_{A}(G)$ of the group $G$, with set of vertices $\pi(G)$, as follows: Two vertices $p, q \in \pi(G)$ form an edge of $\Gamma_{A}(G)$ if either $q \in \pi\left(A_{p}(G)\right)$ or $p \in \pi\left(A_{q}(G)\right)$. It is convenient to write $p \rightarrow q$ if $q \in$ $\pi\left(A_{p}(G)\right)$. For almost simple groups, that is, for groups with a unique minimal normal subgroup, which is a non-abelian simple group, the following result is obtained in the paper.

Main Theorem. Let $G$ be an almost simple group. Then the graph $\Gamma_{A}(G)$ is connected and has diameter at most 5 .

It follows from this theorem that often (with few exceptions) the normalizer of a Sylow $p$-subgroup $G_{p}$ of a finite almost simple group $G$ is larger than $G_{p} C_{G}\left(G_{p}\right)$, for $p$ an odd prime. In particular this is always true if $G$ is a finite simple group (see $[17,16]$ ). However, we obtain here more precise information in graph terms for almost simple groups.

The study of the Sylow graph of a group was first motivated in relation with the influence of the Sylow normalizers (normalizers of the Sylow subgroups) on the structure of finite groups. In Section 5 we show how the result on the Sylow graph of a group can be applied to derive some information about the group within this framework. Examples 1, 2 and 3 show certain classes of groups, which can be considered as extensions of nilpotent groups, and which have the property that a group belongs to the class if and only if its Sylow normalizers belong to the class (Corollaries 5.6 and 5.8). For the classes in Example 1 the problem has been studied in [11] under the assumption of the result on the Sylow graph of a group as a conjecture; though it appears to be a gap in the proof. 
Our results extend some theorems of Glauberman [14], Bianchi et al. [4], and D'Aniello et al. [12]. The generalization in [4] of a result in [14] states that a group is nilpotent whenever its Sylow normalizers are nilpotent. In [12] a similar result is obtained for classes of groups with nilpotent Hall subgroups for given sets of primes, in the universe of all finite soluble groups. In fact, within this universe, this result provides a characterization of the subgroup-closed saturated formations whose elements are characterized by the Sylow normalizers belonging to the class. The corresponding classes are called covering-formations of soluble groups (see Definition in Section 5).

Covering-formations form a special family of the so-called nilpotent-like Fitting formations. These are non-empty saturated Fitting formations locally defined by $\mathcal{E}_{\pi(p)}$, the class of all finite $\pi(p)$-groups for a set of primes $\pi(p)$ containing $p$, for every prime $p$ in the characteristic of the formation. Different restrictions on the sets of primes $\pi(p)$ provide extensions of the nilpotent groups from different points of view. We refer to BallesterBolinches et al. [3] for an account of this development. The classes of groups in Examples 1, 2 and 3 are particular types of covering-formations.

\section{Preliminaries}

Here we collect some lemmas which will be useful in the sequel.

Let $n$ be a positive integer and $p$ a prime number. A prime $r$ is said to be primitive with respect to the pair $\{p, n\}$ if $r$ divides $p^{n}-1$ but $r$ does not divide $p^{e}-1$ for every integer $e$ such that $1 \leq e<n$. It was proved by Zsigmondy [21] that such a primitive prime $r$ exists unless either $n=2$ and $p$ is a Mersenne prime or $\{p, n\}=\{2,6\}$. Moreover, it is well-known that for this prime $r$, it holds $r-1 \equiv 0(\bmod n)$ and, in particular, $r \geq n+1$.

Lemma 2.1. (Frattini argument) Let $G$ be a group, $N$ be a normal subgroup of $G$ and $S$ be a Sylow subgroup of $N$. Then $G=N N_{G}(S)$.

For notation in this section we refer to [7]. Let $G$ be a connected simple algebraic group with root system $\Theta=\Theta(G)$ over an algebraically closed field of characteristic $r>0$. For every simple group $L$ of Lie type defined over the field $\operatorname{GF}\left(r^{t}\right)$, split or twisted, there exists $G=G_{a d}$ and a Frobenius map $\sigma$ of $G$ such that $L=O^{r^{\prime}}\left(G_{\sigma}\right)$, where $G_{\sigma}=\{x \in G \mid \sigma(x)=x\}$. The inner-diagonal automorphism group $\hat{L}$ of $L$ coincides with $G_{\sigma}$. If $G$ has a root system $\Theta$, we say that $L$ is of type $\Theta$ and write $L=\Theta\left(r^{t}\right)$ (see also [8]). 
Lemma 2.2. [17, Theorem 3.3] Let $L=O^{r^{\prime}}\left(G_{\sigma}\right)$ have a root system $\Theta \neq$ $A_{l}(l>1), D_{2 l+1}, E_{6}$. Then every semisimple element of $L$ of odd prime order is conjugate to its inverse, i.e., it is real.

In view of Lemma 2.2 we will call a group of Lie type fine if it has no root system in the set $\left\{A_{l}(l>1), D_{2 l+1}, E_{6}\right\}$.

Lemma 2.3. A field automorphism of a finite simple group of Lie type centralizes the Weyl subgroup of the group modulo its Cartan subgroup.

Proof. We use some facts from [18]. Let $L$ be a finite simple group of Lie type $\Theta$ over the field $F:=\operatorname{GF}(q)$. $L$ has a generating system of elements of the form $x_{\alpha}(\lambda)$ with $\alpha \in \Theta, \lambda \in F$. The elements $w_{\alpha}(\lambda)=$ $x_{\alpha}(\lambda) x_{-\alpha}\left(-\lambda^{-1}\right) x_{\alpha}(\lambda)$ generate the monomial subgroup $N$ and the elements of the form $h_{\alpha}(\lambda)=w_{\alpha}(\lambda) w_{\alpha}(1)^{-1}$ generate the Cartan subgroup $H$ of $L$, which is normal in $N$. Moreover there exists a coset representatives of $H$ in $N$ consisting of elements $\omega_{\alpha}=w_{\alpha}(1)$. If $\phi$ is an automorphism of the field $F$, then the action of the extension of $\phi$ on $L$ can be described as $x_{\alpha}(\lambda)^{\phi}=x_{\alpha}\left(\lambda^{\phi}\right)$. It is easy to see that $\omega_{\alpha}^{\phi}=\omega_{\alpha}$, which proves the result.

Lemma 2.4. Let $L$ be a simple group of Lie type over a field of characteristic $r>0$ and let $P$ be a Sylow $p$-subgroup of $L$, for a prime $p \neq r$. Then:

(a) $P$ normalizes some maximal torus of $L$.

(b) If $p$ does not divide the order of the Weyl group of $L$, then $P$ is contained in some maximal torus of $L$ and hence $P$ is abelian.

Proof. See [5, Corollary II.5.19].

Lemma 2.5. ([6], [7]) Let $L$ be a simple group of Lie type and let $W$ be its Weyl group. Then:

If $L$ is of type $A_{n}$, then $W \cong S_{n+1}$ (of order $(n+1)$ !).

If $L$ is of type $D_{n}$, then $W$ is a semidirect product of an elementary abelian 2-group of order $2^{n}$ and a symmetric group $S_{n}$.

If $L$ is of type ${ }^{2} D_{n}$, then $W$ is a semidirect product of an elementary abelian 2-group of order $2^{n-1}$ and a symmetric group $S_{n-1}$.

If $L$ is of type $E_{6}$, then $W$ has a subgroup of index 2 isomorphic to $P \Omega_{6}^{-}(2) \cong P S p_{4}(3)$.

If $L$ is of type ${ }^{2} E_{6}$, then $W$ is isomorphic to the Weyl group of $F_{4}$ of order $2^{7} 3^{2}$. 


\section{Properties of the graph $\Gamma(G)=\Gamma_{A}(G)$}

We write $\Gamma(G)$ instead of $\Gamma_{A}(G)$ for brevity, as only this type of graphs are studied in the paper. The set of edges of $\Gamma(G)$ is denoted by $\mathrm{E}(\Gamma(G))$. For a group $G$ and a prime $p, \operatorname{Syl}_{p}(G)$ denotes the set of all Sylow $p$-subgroups of the group $G$.

Lemma 3.1. Let $G_{0}$ be a normal subgroup of a group $G$ such that $\left|G / G_{0}\right|=$ $p \in \pi(G)$ and let $r$ be a prime, $r \neq p$. If $r \rightarrow s \in \mathrm{E}\left(\Gamma\left(G_{0}\right)\right)$, then $r \rightarrow s \in$ $\mathrm{E}(\Gamma(G))$.

Proof. Let $R$ be a Sylow $r$-subgroup of $G_{0}$. Then $\left|A_{r}\left(G_{0}\right)\right| \equiv 0(\bmod s)$. It follows from Lemma 2.1 that $\left|N_{G}(R)\right|=\left|N_{G_{0}}(R)\right| p$ and $s \in \pi\left(A_{r}(G)\right)$.

Lemma 3.2. Let $G$ be a group and $P \in \operatorname{Syl}_{p}(G), p>2$. If there exists an element $1 \neq z \in Z(P)$ which is real in $G$, then $p \rightarrow 2 \in \mathrm{E}(\Gamma(G))$.

Proof. The extended centralizer of $z$ in $G$ is defined to be

$$
H:=\left\{y \in G \mid z^{y}=z^{ \pm 1}\right\} .
$$

Clearly $H$ is a subgroup of $G$ and $\left|H: C_{G}(z)\right|=2$. Since $P \leq C_{G}(z)<H$ it is obvious that $\left|A_{p}(H)\right| \equiv 0(\bmod 2)$. Moreover $\operatorname{Syl}_{p}(H) \subseteq \operatorname{Syl}_{p}(G)$ implies that $\pi\left(A_{p}(H)\right) \subseteq \pi\left(A_{p}(G)\right)$ and then the result follows.

For a group $G$, define the distance $\rho(p, q)$, for primes $p, q \in \pi(G)$, as the minimal number $k$ for which there is a chain $p_{0}=p, p_{1}, \ldots, p_{k}=q$ such that every pair $\left(p_{i}, p_{i+1}\right)$ forms an edge. The largest $\rho(p, q), p, q \in \pi(G), p \neq q$, is the diameter of $\Gamma(G)$, denoted by $\mathrm{d}(\Gamma(G))$.

Corollary 3.3. The graph $\Gamma\left(S_{n}\right)$ of a symmetric group of degree $n>2$ is connected and $\mathrm{d}\left(\Gamma\left(S_{n}\right)\right) \leq 2$.

Proof. This follows by Lemma 3.2 since each element of a symmetric group is real.

Lemma 3.4. Let $G$ be an almost simple group and $N$ be a non-trivial normal subgroup of $G$ such that $(|G / N|,|N|)=1$. Then

$$
\mathrm{d}(\Gamma(G)) \leq \mathrm{d}(\Gamma(N))+2 .
$$

If for each pair of different primes $r, s \in \pi(G / N)$ there exists $p \in \pi(N)$ such that rs divides $\left|A_{p}(G)\right|$, then

$$
\mathrm{d}(\Gamma(G)) \leq \mathrm{d}(\Gamma(N))+1 .
$$


Proof. Suppose that $r \in \pi(G) \backslash \pi(N)$. By Lemma 2.1 we have that $|G|_{r}$ divides $\left|N_{G}\left(G_{p}\right)\right|$ for any prime $p \in \pi(N)$ and $G_{p} \in \operatorname{Syl}_{p}(G)$. Since $G$ is almost simple, it is clear that $|G|_{r}$ does not divide $\left|C_{G}\left(G_{p}\right)\right|$ for some prime $p \in \pi(N)$, and then $p \rightarrow r \in \mathrm{E}(\Gamma(G)$. Hence, if $r, s \in \pi(G) \backslash \pi(N)$, it follows that $\rho(r, s) \leq \mathrm{d}(\Gamma(N))+2$.

If $r s$ divides $\left|A_{p}(G)\right|$ for a prime $p \in \pi(N)$, then $\mathrm{d}(\Gamma(G)) \leq \mathrm{d}(\Gamma(N))+1$, since $\rho(r, p)=1$ and $\rho(s, p)=1$.

\section{Proof of the Main Theorem}

Corollary 3.3 is a first step towards the proof of our Main Theorem, which states that the Sylow graph of an almost simple group is connected and has diameter at most 5 . In the next results we consider separately different cases, depending on the simple group appearing as the socle of an almost simple group, and prove that in all cases the desired result holds.

\section{Alternating and sporadic groups}

Theorem 4.1. Let $G=A_{n}$ be an alternating group of degree $n \geq 5$. Then the graph $\Gamma(G)$ is connected and $\mathrm{d}(\Gamma(G)) \leq 3$. If $n \neq p, p+1$, with $p$ a prime, $p \equiv 3(\bmod 4)$, then $\mathrm{d}(\Gamma(G))=2$.

Proof. Let $p$ be a prime, $3 \leq p \leq n$. Assume that $p \rightarrow 2 \notin \mathrm{E}(\Gamma(G))$. By Lemma 3.2 there exists an element $z$ of order $p$ which is not real in $G$. If $p \leq n-2$, then $z=a_{1} a_{2} \ldots a_{k}$ where $a_{1}, a_{2}, \ldots, a_{k}$ are disjoint cycles of length $p, k \geq 1$ and $k p+r=n$.

Suppose first that $r \geq 2$. In this case there exists an odd permutation $\sigma$ centralizing $z$. On the other hand, $z^{g}=z^{-1}$ for some $g \in S_{n}$. If $g \in S_{n} \backslash A_{n}$, then $z^{\sigma g}=z^{-1}$ and $\sigma g \in A_{n}$. But this is a contradiction by Lemma 3.2. Hence $r \leq 1$.

If $k>1$, then there exists an involution $\sigma \in S_{n} \backslash A_{n}$ permuting $a_{1}$ and $a_{2}$ and fixing all letters not appearing in $a_{1}$ and $a_{2}$. This gives a contradiction as above since $z^{\sigma}=z$ and $z^{\sigma g}=z^{-1}$.

Assume that either $n=p$ or $n=p+1$. There is an element $g \in$ $S_{n}$ inverting $z$ which is a product of $\frac{p-1}{2}$ transpositions $g=(1, p)(2, p-$ 1) $\ldots\left(\frac{p-1}{2}, \frac{p+3}{2}\right)$, as a permutation of the set $\{1,2, \ldots, n\}$. Since $g \in S_{n} \backslash A_{n}$, we have $\frac{p-1}{2} \equiv 1(\bmod 2)$, which implies $p \equiv 3(\bmod 4)$.

In this case there exists an odd prime $s$ dividing $p-1$. It is easy to see that $p \rightarrow s \in \mathrm{E}(\Gamma(G))$ and $s \rightarrow 2 \in \mathrm{E}(\Gamma(G))$ by using arguments as above. 
Therefore for each pair $p, r \in \pi(G)$ there is a path from $p$ to $r$ of length at most 3. The theorem is proved.

Remark. The alternating group $A_{8}$ shows that the estimate of Theorem 4.1 is sharp. In this case $\pi\left(A_{8}\right)=\{2,3,5,7\}$ and $\mathrm{E}\left(\Gamma\left(A_{8}\right)\right)=\{7 \rightarrow 3,3 \rightarrow$ $2,5 \rightarrow 2\}$.

Theorem 4.2. Let $L$ be a sporadic simple group and $L \leq G \leq \operatorname{Aut}(L)$. Then the graph $\Gamma(G)$ is connected and $\mathrm{d}(\Gamma(G)) \leq 5$.

Proof. This is easy to prove using the description in [15] of the normalizers of Sylow subgroups of the considered groups.

Observe that there are only 3 sporadic simple groups whose Sylow graphs have the diameter exactly 5 . They are the Mathieu group $M_{23}$, the Baby Monster group $B$ and the Monster group $M$.

\section{Almost simple groups with fine socle}

We recall that a group of Lie type is called fine if it has no root system in the set $\left\{A_{l}(l>1), D_{2 l+1}, E_{6}\right\}$.

Theorem 4.3. Let $G$ be an almost simple group whose socle is a fine group of Lie type. Then the graph $\Gamma(G)$ is connected and $\mathrm{d}(\Gamma(G)) \leq 4$.

Proof. Let $L$ be a fine group of Lie type over the field $\operatorname{GF}\left(r^{t}\right)$ and set $G_{0}=G \cap \operatorname{Inndiag}(L)$.

Note that, by Lemma 2.2, for a group $X$ such that $L \leq X \leq G$ every semisimple element of odd prime order in $L$ is real. Suppose that $p$ is an odd prime in $\pi(L) \backslash\{r\}$. Then a Sylow $p$-subgroup $L_{p}$ of $L$ is normal in some Sylow $p$-subgroup $X_{p}$ of $X$ and there exists an element $1 \neq z \in Z\left(X_{p}\right) \cap L_{p}$ such that $z$ is conjugate with $z^{-1}$. By Lemma 3.2 we have that $p \rightarrow 2 \in$ $\mathrm{E}(\Gamma(X))$.

Assume first that the characteristic $r$ of $L$ is odd.

Let $L \neq L_{2}(q)$ with $q \equiv 3(\bmod 4)$. By the above property and inspecting the orders of the Borel subgroups of $L$, we have that $p \rightarrow 2 \in \mathrm{E}\left(\Gamma\left(G_{0}\right)\right)$ for any odd prime $p \in \pi\left(G_{0}\right)$. In particular $\mathrm{d}\left(\Gamma\left(G_{0}\right)\right) \leq 2$.

This fact also holds for any group $G_{1} \geq G_{0}$ such that $\pi\left(G_{1}\right)=\pi\left(G_{0}\right)=$ $\pi(L)$. Hence we consider $G_{1} \triangleleft G$ such that $\pi\left(G / G_{1}\right) \cap \pi(L)=\emptyset$.

We claim that $\mathrm{d}(\Gamma(G)) \leq 3$. Now we may apply Lemma 3.4. Since the graph automorphism of $L$ does not centralize any Sylow $r$-subgroup of $G_{1}$, we have that $\mathrm{d}(\Gamma(G)) \leq \mathrm{d}\left(\Gamma\left(G_{1}\right)\right)+1 \leq 3$. 
Let now $L=L_{2}(q)$ where $q \equiv 3(\bmod 4)$. It is well-known that the normalizer of a Sylow $r$-subgroup of $L$ is a Frobenius group of order $\frac{q(q-1)}{2}$, and the centralizer of any field automorphism of $L$ does not contain a Sylow $r$-subgroup of $L$. If $|G / L| \equiv 0(\bmod 2)$, then again $r \rightarrow 2 \in \mathrm{E}(\Gamma(G))$. Clearly, if $v \in \pi(t) \cap(\pi(G) \backslash\{r\})$, then also $r \rightarrow v$. It is obvious that $\mathrm{d}(\Gamma(G)) \leq 3$ if $P G L_{2}(q)$ is a subgroup of $G$, and $\mathrm{d}(\Gamma(G)) \leq 4$ if $|G / L|$ is odd.

The case $L=L_{2}(q)$ with $q=3^{3^{a}}$ demonstrates the following interesting phenomena. If $G$ is an extension of $L$ by the group of order dividing $3^{a}>1$, then the Sylow 3-subgroups of $G$ are self-normalizing (see, for instance, [16]). If $K$ is the normalizer in $G$ of a Sylow 2-subgroup of $G$ (and of $L$ ), then $K / O(K) \cong A_{4}$, the alternating group of degree 4 , and we have $2 \rightarrow 3$. In this case $\mathrm{d}(\Gamma(G))=2$. In the case when 2 divides the order of $G / L$, we have also $\mathrm{d}(\Gamma(G))=2$, but the structure of the graph $\Gamma(G)$ is slightly different.

Now consider the case when $r=2$ and $t>1$.

Again, it follows as above that $p \rightarrow 2 \in \mathrm{E}\left(\Gamma\left(G_{1}\right)\right)$, for each odd prime $p \in \pi(L)$, and a group $G_{1}$ such that $G_{0} \leq G_{1} \unlhd G, \pi\left(G_{1}\right)=\pi\left(G_{0}\right)=\pi(L)$ and $\pi\left(G / G_{1}\right) \cap \pi(L)=\emptyset$. In particular, $\mathrm{d}\left(\Gamma\left(G_{1}\right)\right) \leq 2$.

Suppose that $p \in \pi(G) \backslash \pi(L)$. This is the case only when $p$ is the order of some field automorphism $\varphi$ of $L$. Since $\varphi$ centralizes no Sylow 2-subgroup of $L$, it follows from Lemma 3.4 that $\mathrm{d}(\Gamma(G)) \leq \mathrm{d}\left(\Gamma\left(G_{1}\right)\right)+1 \leq 3$, and the theorem is proved.

\section{Almost simple groups with socle $L=E_{6}^{ \pm}(q)$}

In Lemmas $4.4-4.7$ and Theorem $4.8, L$ is $E_{6}^{ \pm}(q), r$ denotes the characteristic of $L$ and $W(L)$ its Weyl group. Here $E_{6}^{+}(q)$ and $E_{6}^{-}(q)$ stand for $E_{6}(q)$ and ${ }^{2} E_{6}(q)$, respectively. It is convenient to write $L=E_{6}^{\epsilon}=E_{6}^{ \pm}, \epsilon= \pm$.

If $d=(3, q-\epsilon 1)$, then the order of $L$ is as follows:

$$
\begin{aligned}
|L|=\left|E_{6}^{ \pm}(q)\right|= & \frac{1}{d} q^{36}(q-\epsilon 1)^{6}(q+\epsilon 1)^{4}\left(q^{2}-\epsilon q+1\right)^{2}\left(q^{2}+\epsilon q+1\right)^{3} \\
& \left(q^{2}+1\right)^{2}\left(q^{4}-q^{2}+1\right)\left(q^{4}+1\right) \frac{q^{5}-\epsilon 1}{q-\epsilon 1}\left(q^{6}+\epsilon q^{3}+1\right) .
\end{aligned}
$$

It was proved by Stensholt in $[19,20]$ that $F_{4}(q)$ is a subgroup of $L$. The order of the Weyl group of $F_{4}(q)$ is $2^{7} 3^{2}$, and all semisimple elements of odd prime order in $F_{4}(q)$ are real by Lemma 2.2, since this is a fine group.

Lemma 4.4. If $s \geq 5$ is a prime dividing $q^{4}-q^{2}+1, q^{2} \pm q+1, q^{2}+1$ or $q^{4}+1$, then $s \rightarrow 2 \in \mathrm{E}(\Gamma(L))$. 
Proof. If $s>5$ the Sylow $s$-subgroups of $L$ are abelian by Lemma 2.4.

Consider first the case $L=E_{6}^{+}(q)$. Since all semisimple elements of odd prime order in $F_{4}(q)$ are real, we have that $s \rightarrow 2 \in \mathrm{E}(\Gamma(L))$ by Lemma 3.2.

Suppose that $1 \neq R \in \operatorname{Syl}_{5}(L)$. Then 5 divides $q^{2}+1$. In this case $R$ normalizes a torus $T$ such that $\left|N_{L}(T) / T\right| \equiv 0(\bmod 5)$. It follows from [9] that there is just one such torus $D_{4}\left(a_{1}\right)$ and its index in the normalizer is 96. Hence the Sylow 5-subgroups are abelian and $5 \rightarrow 2 \in \mathrm{E}(\Gamma(L))$.

Let now $L=E_{6}^{-}(q)$. Since the Weyl group of $L$ is of type $F_{4}$ of order $2^{7} 3^{2}$, it follows that every Sylow $s$-subgroup is abelian, and since $s \in \pi\left(F_{4}(q)\right)$, every element of order $s$ in $L$ is real. Hence $s \rightarrow 2 \in \mathrm{E}(\Gamma(L))$ as asserted.

Lemma 4.5. If $s \neq 5$ is a prime dividing $q^{4}+\epsilon q^{3}+q^{2}+\epsilon q+1$ and not dividing $q-\epsilon 1$, then $s \rightarrow 5 \in \mathrm{E}(\Gamma(L))$.

Proof. It is easy to see that $s \mid q^{5}-\epsilon 1$ and all other factors $q^{i} \pm 1$ dividing $|L|$ have common prime divisors with $q^{5}-\epsilon 1$ only in $\pi(q-\epsilon 1)$. By Lemma 2.4 the Sylow $s$-subgroups of $L$ are abelian.

Consider first the case $L=E_{6}^{+}(q)$. By [9] there are tori $T$ of types $A_{4}$ and $A_{4}+A_{1}$ of $L$, of orders $\frac{\left(q^{5}-1\right)(q-1)}{(q-1,3)}$ and $\frac{\left(q^{5}-1\right)(q+1)}{(q-1,3)}$, respectively. In both cases $\left|N_{L}(T): T\right|=10$.

Note that $L$ contains a section $K$ isomorphic to $L_{2}(q) \times L_{5}(q)$, which follows from the Dynkin diagram of this group. $K$ has a Frobenius subgroup of order $\frac{q^{5}-1}{(q-1)(q-1,5)} \cdot 5$ and the Sylow $s$-subgroups of $L$ are cyclic. Hence there is an edge $s \rightarrow 5 \in \mathrm{E}(\Gamma(L)$.

In the case $L=E_{6}^{-}(q)$, the group $L$ contains a section $K$ isomorphic to $L_{2}(q) \times U_{5}(q)$ and $U_{5}(q)$ has a Frobenius subgroup of order $5\left(q^{4}-q^{3}+q^{2}-\right.$ $q+1)$. Again the Sylow $s$-subgroups of $L$ are cyclic. Hence there is an edge $s \rightarrow 5 \in \mathrm{E}(\Gamma(L)$, which concludes the proof.

Lemma 4.6. $5 \rightarrow 2 \in \mathrm{E}(\Gamma(L))$; if $r \neq 2$, then $r \rightarrow 2 \in \mathrm{E}(\Gamma(L))$.

Proof. It follows from the description of the Borel and Cartan subgroups of $L$ that $r \rightarrow 2 \in \mathrm{E}(\Gamma(L))$ if $r \neq 2$. Hence we may assume that $5 \neq r$ and 5 divides either $q^{2}-1$ or $q^{2}+1$. By Lemma 4.4 we need only to consider the case when 5 divides either $q-1$ or $q+1$. By Lemma 2.4 the Sylow 5 -subgroups of $E_{6}^{-}(q)$ are abelian. Since $5 \in \pi\left(F_{4}(q)\right)$ there is nothing to prove in this case.

Consider the case $E_{6}^{+}(q)$. Assume first that 5 divides $q+1$. Then a Sylow 5-subgroup of $L$ is a Sylow 5-subgroup of $F_{4}(q)$. Since each element of order 5 of $F_{4}(q)$ is real, we have $5 \rightarrow 2 \in \mathrm{E}(\Gamma(L))$. 
Suppose now that 5 divides $q-1$. Then the order of a Sylow 5 -subgroup of $L$ is $5(q-1)_{5}^{6}$. By $[9]$ there exists a torus $T$ of order $\frac{(q-1)^{6}}{(q-1,3)}$ with $N_{L}(T) / T \cong$ $W(L)$ of order $2^{7} 3^{4} 5$. Since $W(L)$ is generated by involutions, we have that $W(L)$ has no normal 5-complement and, in this case, the normalizer of a Sylow 5-subgroup $S$ of $W(L)$ contains a 2-element acting non-trivially on $S$. Hence $2 \in \pi\left(A_{5}(L)\right)$ and $5 \rightarrow 2 \in \mathrm{E}(\Gamma(L))$, as asserted.

Lemma 4.7. $3 \rightarrow 2 \in \mathrm{E}(\Gamma(L))$ and $\mathrm{d}(\Gamma(L)) \leq 4$.

Proof. Prove first that $3 \rightarrow 2 \in \mathrm{E}(\Gamma(L))$. By Lemma 4.6 we may assume that $r \neq 3$.

Suppose that $q \equiv-\epsilon 1(\bmod 3)$. Then the order of a Sylow 3 -subgroup of $L$ is $3^{2}(q+\epsilon 1)_{3}^{4}$. Since $F_{4}(q)$ is a subgroup of $L$, we have that every element of order 3 of $L$ is real and $3 \rightarrow 2 \in \mathrm{E}(\Gamma(L))$ by Lemma 3.2.

Now let $q \equiv \epsilon 1(\bmod 3)$. Then the order of a Sylow 3 -subgroup of $L$ is $3^{3}(q-\epsilon 1)_{3}^{6}$. In this case there is a torus $T$ of $L$ of order $(q-\epsilon 1)^{6} /(q-\epsilon 1,3)$ and the Sylow 3-subgroup of $T$ is normalized by $W(L)$. It is easy to see that $W(L)$ has self-centralizing Sylow 3-subgroups and $2 \in \pi\left(A_{3}(W)\right)$. By Lemma 2.1 we obtain that $3 \rightarrow 2 \in \mathrm{E}(\Gamma(L))$.

We claim that $p \rightarrow 2 \in \mathrm{E}(\Gamma(L))$ for every prime $p \in \pi\left(q^{2}-1\right)$. By Lemmas 4.4, 4.6 and the previous arguments we may assume that $p \geq 7$. It follows from Lemma 2.4 that the Sylow $p$-subgroups of $L$ are abelian. Since the elements of order $p$ in $F_{4}(q)$ are real, the claim follows by Lemma 3.2.

Consider now a prime $p \in \pi\left(\frac{q^{6}+\epsilon q^{3}+1}{(3, q-\epsilon 1)}\right)$. It follows from [9] and the description of the centralizers of the elements of order 3 in L (see lecture by Iwahori in [5], and [2]) that there is a Frobenius subgroup of order $9\left(q^{6}+\right.$ $\left.\epsilon q^{3}+1\right)$. Hence $p \rightarrow 3$.

Whence, from Lemmas $4.4-4.6$, it follows now that $\mathrm{d}(\Gamma(L)) \leq 4$ and the lemma is proved.

Theorem 4.8. Let $G$ be an almost simple group with socle $L=E_{6}^{ \pm}(q)$. Then the graph $\Gamma(G)$ is connected and $\mathrm{d}(\Gamma(G)) \leq 5$.

Proof. By Lemma 2.3 the field automorphisms of $L$ centralize $W(L)$ and, therefore, for each semisimple element of order $p$ in $L$, if some edge $p \rightarrow s$ exists in $\Gamma(L)$, then $p \rightarrow s \in \mathrm{E}(\Gamma(G))$. If $r \neq 3$, then the edge $r \rightarrow 2$ also remains in $\Gamma(\operatorname{Autdiag}(L))$.

Suppose that $\varphi$ is a field automorphism of $L$ of order coprime to $r$. This automorphism preserves all edges of the form $p \rightarrow s$ if $p \neq r$. On the other hand, if $r \neq 2$ it is clear that the edge $r \rightarrow 2$ remains in the automorphism group. If $r=2$ we are done by Lemma 3.1. Now suppose that $\varphi$ is an 
automorphism of $L$ of order coprime to $|L|$. Then, as in Lemma 3.4, it follows that $d(G) \leq d(L)+1 \leq 5$.

Almost simple groups with socle $L=P \Omega_{4 l+2}^{\epsilon}(q)$

Theorem 4.9. Let $G$ be an almost simple group with socle $L$ isomorphic to a group $P \Omega_{4 l+2}^{\epsilon}(q)\left(\epsilon= \pm, q=r^{t}, l \geq 1\right)$. Then the Sylow graph $\Gamma(G)$ is connected and $\mathrm{d}(\Gamma(G)) \leq 5$.

Proof. Define the set $\sigma_{1} \subseteq \pi(L)$ as follows:

If $\varepsilon=+, \sigma_{1}=\{p \mid p$ a prime primitive with respect to the pair $(q, 2 l+1)\}$; if $\epsilon=-, \sigma_{1}=\{p \mid p$ a prime primitive with respect to the pair $(q, 4 l+2)\}$. Set also $\sigma_{3}:=\{r, 2\}$ and $\sigma_{2}:=\left(\pi(L) \backslash \sigma_{1}\right) \backslash \sigma_{3}$. By [19] we have that $L$ has a subgroup $M$ isomorphic to $P \Omega_{4 l}^{\epsilon}(q)$ and, moreover, $\pi(L)=\pi(M) \cup \sigma_{1}$.

We prove next that for each odd prime $p \in \sigma_{2}$ there is an edge $p \rightarrow 2 \in$ $\mathrm{E}(\Gamma(L))$. Clearly this will be also true for $\Gamma(\operatorname{Inndiag}(L))$ instead of $\Gamma(L)$ by Lemma 3.1.

By Lemma 2.2, since $M$ is a fine group, every semisimple element of odd prime order in $M$ is real. Let $p \in \sigma_{2}$. Since in this case $p \in \pi(M)$ and a Sylow $p$-subgroup of $M$ is a Sylow $p$-subgroup of $L$, this implies that $p \rightarrow 2 \in E(\Gamma(L))$.

We prove now that for each $p \in \sigma_{1}$ there exists a prime $s \in \sigma_{2}$ such that $p \rightarrow s \in \mathrm{E}(\Gamma(L))$. It is clear from the Dynkin diagram of $L$ that $A_{2 l} \subset D_{2 l+1}$. Hence the group $P \Omega_{4 l+2}^{+}(q)$ contains the normalizer of a Singer subgroup of $L_{2 l+1}(q)$. This means that for each prime $p$ primitive with respect to the pair $(q, 2 l+1)$ and each prime divisor $s$ of $2 l+1$ there is an edge $p \rightarrow s \in \mathrm{E}(\Gamma(L))$. Clearly, $s \in \sigma_{2}$. Since $P \Omega_{4 l+2}^{-}(q)$ contains $U_{2 l+1}(q)$, we can deduce as before that for every prime $p$ primitive with respect to the pair $(q, 4 l+2)$ and $s \in \pi(2 l+1)$, there exists an edge $p \rightarrow s \in \mathrm{E}(\Gamma(L))$.

If $r>2$, the order of the Borel subgroup of $L$ is divisible by any prime in $\pi(q-1)$ and then $r \rightarrow 2 \in \mathrm{E}(\Gamma(L))$.

It follows from Lemma 3.1 that the graphs $\Gamma(L)$ and $\Gamma(\operatorname{Inndiag}(L))$ are connected and have diameter at most 4 .

Let $G_{0} \geq L$ be a maximal normal subgroup of $G$ such that $\pi\left(G_{0}\right)=\pi(L)$. Clearly, $\operatorname{Inndiag}(L) \leq G_{0}$. Since the field automorphisms of $L$ centralize the Weyl group of $L$ (Lemma 2.3), all edges of the form $p \rightarrow s$ for primes in $\sigma_{1} \cup \sigma_{2}$ are also in $\mathrm{E}\left(\Gamma\left(G_{0}\right)\right)$. Moreover $r \rightarrow 2 \in \mathrm{E}\left(\Gamma\left(G_{0}\right)\right)$ because every field automorphism has fixed points on the Cartan subgroup of $L$ of order at least $\frac{1}{d}(r-1)^{2 l}$. Therefore $\mathrm{d}\left(\Gamma\left(G_{0}\right)\right) \leq \mathrm{d}(\Gamma(L)) \leq 4$. Now we deduce from Lemma 3.4 that $\mathrm{d}(\Gamma(G)) \leq 5$. 


\section{Almost simple groups with socle $L=L_{l}^{\epsilon}(q)$}

Theorem 4.10. Let $G$ be an almost simple group with socle $L$ isomorphic to $L_{l}^{\epsilon}(q)\left(\epsilon= \pm, q=r^{t}, l>2\right)$. Then the Sylow graph of $G$ is connected and $\mathrm{d}(\Gamma(G)) \leq 5$.

Proof. Define the set $\sigma_{1} \subseteq \pi(L)$ as follows:

If $\epsilon=+, \sigma_{1}$ is defined to be the set of primes primitive with respect to the pair $(q, j)$ for $j \leq l<2 j$.

If $\epsilon=-, \sigma_{1}$ is the set of primes $p$ such that $p \mid q^{j}-(\epsilon 1)^{j}(j \leq l<2 j)$ and $p$ is primitive with respect to the pair $(q, j)$ when $j$ is even, and with respect to the pair $(q, 2 j)$ when $j$ is odd.

Set also $\sigma_{3}:=\{r, 2\}$ and $\sigma_{2}:=\left(\pi(L) \backslash \sigma_{1}\right) \backslash \sigma_{3}$.

We prove first that $p \rightarrow 2 \in \mathrm{E}(\Gamma(L))$ for any odd prime $p \in \sigma_{2} \cup \sigma_{3}$.

If $p=r$, any Sylow $r$-subgroup of $L$ contains its centralizer in Inndiag $(L)$. Hence $r \rightarrow s \in \mathrm{E}(\Gamma(G))$ for every $s \in \pi(r-1)$. In particular this holds for $s=2$ and $r>2$.

If $p \in \sigma_{2}$, then $p$ divides $q^{i}-(\epsilon 1)^{i}$ for some $i$, which is minimal with this property. We consider $l=m i+\lambda$ for some $0 \leq \lambda<i$. In this case $m>1$ by the definition of $\sigma_{2}$ and there exists a subgroup $R$ of $K=G L_{l}^{\epsilon}(q)$ consisting of all matrices $\operatorname{diag}\left(A_{1}, \ldots, A_{m}, I\right)$, where $A_{i} \in G L_{i}^{\epsilon}(q)$ are of order $f(q, p)=\left(q^{i}-(\epsilon 1)^{i}\right)_{p}$ and $I$ is the identity $\lambda \times \lambda$-matrix.

It follows from arithmetical arguments that $|K|_{p}=\left(q^{i}-(\epsilon 1)^{i}\right)_{p}^{m}(m !)_{p}$. The subgroup $R$ is a direct product of $m$ copies of groups of order $f(q, p)$. Obviously the subgroup $S_{m}$ permuting blocks of the matrices of size $i$ is contained in $S L_{l}^{\epsilon}(q)$ and normalizes $R$. Moreover, this subgroup contains a Sylow $p$-subgroup of $K$. Now we can deduce from Corollary 3.3 that $2 \in \pi\left(A_{p}(L)\right)$ and so $p \rightarrow 2 \in \mathrm{E}(\Gamma(L))$.

We claim that for every $p \in \sigma_{1}$ there exists an edge $p \rightarrow s$ for some $s \in \sigma_{2} \cup \sigma_{3}$. Let $p \in \sigma_{1}$. If $p \mid q^{j}-(\epsilon 1)^{j}$ with $2 j>l$, then a Sylow $p$ subgroup of $L$ is cyclic and isomorphic to a subgroup of a Singer subgroup of the group $L_{j}^{\epsilon}(q)$. Since $2 j>l$, this implies, from properties of Singer subgroups, that $|L|_{p}=\left(q^{j}-(\epsilon 1)^{j}\right)_{p}$. Moreover, in this case, for every prime divisor $s$ of $j$ there exists an element of order $s$ that normalizes, but not centralizes, a Sylow $p$-subgroup of $L$. Hence there exists an edge $p \rightarrow s$. We see next that $s \in \sigma_{2} \cup \sigma_{3}$. This is clear if either $j \equiv 0(\bmod r)$ or $j \equiv 0(\bmod 2)$. Hence we may assume that $j$ is odd. If $j$ is not a prime, since $s$ divides $j$, we have $s \mid q^{s-1}-1, s \leq \frac{j}{2}$ and hence $2 s \leq j \leq l$. This forces $2(s-1)<l$ and $s \in \sigma_{2}$. Suppose that $j=s$. As above $s \mid q^{s-1}-1$. If $s$ is primitive with respect to the pair $(q, s-1)$, then $s \notin \sigma_{1}$ and so $s \in \sigma_{2} \cup \sigma_{3}$. 
Otherwise, $s \mid q^{i}-1$ for some $i$ dividing $s-1$. Hence $2 i<l$ and $s \in \sigma_{2}$, which proves the claim.

Whence it follows that in fact the group $P G L_{l}^{\epsilon}(q)$ has Sylow graph with diameter at most 4 . We can argue now like in the proof of Theorem 4.9, for $L=P \Omega_{4 l+2}^{ \pm}(q)$, to obtain finally the desired result.

The Main Theorem follows now from Corollary 3.3 and Theorems 4.1, $4.2,4.3,4.8,4.9,4.10$.

\section{An application: Sylow normalizers}

In this section we apply the results on the Sylow graph of a group to obtain some information about the structure of groups.

Definition. ([12, Definition]) A covering-formation $\mathcal{F}=L F(f)$ is a nonempty saturated formation locally defined by a formation function $f$ given by:

$$
f(p)=\mathcal{E}_{\pi(p)} \text { if } p \in \pi, \quad f(q)=\emptyset \text { if } q \notin \pi,
$$

$\pi:=\operatorname{Char}(\mathcal{F})$, the characteristic of $\mathcal{F}$, where for each prime $p \in \pi, \pi(p)$ is a set of primes satisfying the following conditions:

(i) $p \in \pi(p) \subseteq \pi$,

(ii) $q \in \pi(p) \Longrightarrow p \in \pi(q)$,

and where $\mathcal{E}_{\pi(p)}$ is the class of all finite $\pi(p)$-groups.

(As mentioned in [12, Remark 1(b)] the condition $\pi(p) \subseteq \pi$ is no loss of generality.)

Let $\mathcal{S}$ denote the class of all finite soluble groups and let $\mathcal{S}_{\pi(p)}$ be the class of all finite soluble $\pi(p)$-groups. The saturated formation $\mathcal{F} \cap \mathcal{S}=L F(g)$ such that

$$
g(p)=\mathcal{S}_{\pi(p)} \text { if } p \in \pi, \quad g(q)=\emptyset \text { if } q \notin \pi,
$$

is called a covering-formation of soluble groups.

With the previous notation, if $q \in \pi(p)$, we write $p \leftrightarrow q$. (This defines a reflexive and symmetric relation on $\pi$.) We associate to the coveringformation $\mathcal{F}$ another family of sets of primes defined as follows:

$$
\Sigma_{\mathcal{F}}:=\{\sigma \subseteq \pi \mid \text { if } p, q \in \sigma \text { and } p \neq q \text {, then } p \leftrightarrow q\} .
$$

We say that two primes $r$ and $p$ of $\pi$ are connected if either $r=p$ or there is a sequence of primes $r=r_{1}, \ldots, r_{n}=p$ in $\pi$ such that $r_{i} \leftrightarrow r_{i+1}$ for any $1 \leq i \leq n-1$. 
In the sequel $\mathcal{F}$ always denotes a covering-formation as defined above. We introduce some more notation.

For a group $G$ and a prime $p$, let $G_{p}$ denote a Sylow $p$-subgroup of $G$. Recall that $\pi(G)$ denotes the set of all primes dividing the order of $G$. Then, for a class $\mathcal{X}$ of groups, the class map $\mathrm{N}$ is defined as follows:

$$
\mathrm{N} \mathcal{X}=\left(G \mid N_{G}\left(G_{p}\right) \in \mathcal{X}, \text { for every prime } p \in \pi(G)\right) \text {. }
$$

For notation and results about classes of groups and closure operations we refer to Doerk and Hawkes book [13].

We are interested in the study of classes of groups $\mathcal{X}$ satisfying $\mathrm{N} \mathcal{X}=$ $\mathcal{X}$, that is, a group $G$ belongs to such a class $\mathcal{X}$ if and only if its Sylow normalizers belong to the class.

In [12] the covering-formations of soluble groups are characterized as the subgroup-closed saturated formations $\mathcal{X}$ satisfying that $\mathrm{N} \mathcal{X}=\mathcal{X}$ in the universe of all finite soluble groups. Also they are exactly the classes of soluble groups with nilpotent Hall subgroups for prefixed sets of primes. For any set of primes $\tau$ we set $\mathbf{E}_{\tau}^{\mathrm{n}}$ for the class of all groups with nilpotent Hall $\tau$-subgroups. To be more precise we gather the following results.

Proposition 5.1. [12, Proposition 2] Let $\mathcal{G}=\mathcal{F} \cap \mathcal{S}$ be a covering-formation of soluble groups as defined above. Then:

1. $\mathrm{s} \mathcal{G}=\mathcal{G}$ and $\mathrm{N} \mathcal{G} \cap \mathcal{S}=\mathcal{G}$;

2. $\mathcal{G}=\bigcap_{\sigma \in \Sigma_{\mathcal{F}},|\sigma|=2} \mathbf{E}_{\sigma}^{\mathrm{n}} \cap \mathcal{S}=\bigcap_{\sigma \in \Sigma_{\mathcal{F}}} \mathbf{E}_{\sigma}^{\mathrm{n}} \cap \mathcal{S}$.

Theorem 5.2. [12, Theorem] Let $\mathcal{H}$ be a subgroup-closed saturated formation of soluble groups. Then the following statements are equivalent:

(i) For any soluble group, its Sylow normalizers belong to $\mathcal{H}$ if and only if the group belongs to $\mathcal{H}$;

(ii) $\mathcal{H}$ is a covering-formation of soluble groups.

The next technical result will be useful to clarify the constructions in the examples below.

Lemma 5.3. Let $\left\{\tau_{i} \mid i \in I\right\}$ be a partition of a set of primes $\tau$ and let $\mathcal{D}=L F(d)$ be a saturated formation of characteristic $\tau$, locally defined by a formation function d satisfying that $\mathcal{S}_{p} d(p) \subseteq \mathcal{E}_{\tau_{i}}$ for every $i \in I$ and every prime $p \in \tau_{i} \subseteq \tau$. Then

$$
\mathcal{D}=\times_{i \in I} \mathcal{D}_{i}:=\left(G \in \mathcal{E}_{\tau} \mid G=\times_{i \in I} G_{\tau_{i}}, G_{\tau_{i}} \in \mathcal{D}_{i}\right)
$$


where for every $i \in I, \mathcal{D}_{i}:=\mathcal{D} \cap \mathcal{E}_{\tau_{i}}=L F\left(d_{i}\right)$ is the saturated formation of characteristic $\tau_{i}$, locally defined by the formation function $d_{i}$ given by

$$
d_{i}(p)=d(p) \text { if } p \in \tau_{i}, \quad d_{i}(q)=\emptyset \text { if } q \notin \tau_{i} .
$$

Proof. The result is easily proven taking into account that

$$
\begin{gathered}
\mathcal{D}=\mathcal{E}_{\tau} \cap\left(\cap_{p \in \tau} \mathcal{E}_{p^{\prime}} \mathcal{S}_{p} d(p)\right) \subseteq \mathcal{E}_{\tau} \cap\left(\cap_{i \in I} \mathcal{E}_{\tau_{i}^{\prime}} \mathcal{E}_{\tau_{i}}\right)=\left(G \in \mathcal{E}_{\tau} \mid O^{\tau_{i}}(G) \in \mathcal{E}_{\tau_{i}^{\prime}}, i \in I\right)= \\
=\left(G \in \mathcal{E}_{\tau} \mid G=\times_{i \in I} G_{\tau_{i}}, G_{\tau_{i}} \in \mathcal{E}_{\tau_{i}}\right) .
\end{gathered}
$$

Remark. [13, Proposition $\operatorname{IV}(3.8)]$ We recall that if $\mathcal{D}=L F(D)=L F(d)$ is a saturated formation with canonical local definition $D$ and where $d$ is any other local definition of $\mathcal{D}$, then $D(p)=\mathcal{S}_{p} d(p) \cap \mathcal{D}$ for any prime $p$.

Hence the hypothesis on the local definition $d$ in Lemma 5.3 can be equivalently replaced by the assumption that the canonical local definition $D$ of $\mathcal{D}$ satisfies that $D(p) \subseteq \mathcal{E}_{\tau_{i}}$ for every $i \in I$ and every prime $p \in \tau_{i} \subseteq \tau$.

Example 1. Let $\left\{\pi_{i} \mid i \in I\right\}$ be a partition of the set of primes $\pi$ and

$$
\times_{i \in I} \mathcal{E}_{\pi_{i}}:=\left(G \in \mathcal{E}_{\pi} \mid G=\times_{i \in I} G_{\pi_{i}}, G_{\pi_{i}} \in \mathcal{E}_{\pi_{i}}\right) .
$$

As follows from Lemma 5.3, this class is a covering-formation $\mathcal{F}=\times_{i \in I} \mathcal{E}_{\pi_{i}}$ such that the sets of primes $\pi(p)(p \in \pi)$ form the given partition of $\pi$.

Example 1 is a particular type of the following construction:

Example 2. Let $\mathcal{H}$ be a covering-formation of (soluble) groups of characteristic $\rho(2 \notin \rho), \pi=\rho \cup \tau$ and $\rho \cap \tau=\emptyset$. Let

$$
\mathcal{H} \times \mathcal{E}_{\tau}:=\left(G \in \mathcal{E}_{\pi} \mid G=A \times B, A \in \mathcal{H}, B \in \mathcal{E}_{\tau}\right) .
$$

Then $\mathcal{F}=\mathcal{H} \times \mathcal{E}_{\tau}$ is a covering-formation such that if $2, p \in \pi$ and $p$ is connected with 2 , then $\pi(p)=\pi(2)=\tau$. Moreover, $\mathcal{F}=\mathcal{H} \times \mathcal{E}_{\tau}=$ $\bigcap_{\sigma \in \Sigma_{\mathcal{F}}} \mathbf{E}_{\sigma}^{\mathrm{n}}$.

Proof. The first part follows from Lemma 5.3. Then from Proposition 5.1 we can deduce that $\mathcal{F} \subseteq \mathcal{L}:=\bigcap_{\sigma \in \Sigma_{\mathcal{F}}} \mathbf{E}_{\sigma}^{\mathrm{n}}$.

Assume now that $\mathcal{F}$ is properly contained in $\mathcal{L}$ and let $G \in \mathcal{L} \backslash \mathcal{F}$ of minimal order. Since $\mathcal{L}$ is Q-closed and $\mathcal{F}$ is a saturated formation, $G$ is a primitive group of type either 1 or 2 , with a unique minimal normal subgroup, say $N$, and $G / N \in \mathcal{F}$. 
Assume that $G$ is primitive of type 1 and $N$ is a $p$-group for a prime $p$. Since $G \in \mathcal{L}$, it follows that $G / N=G / C_{G}(N) \in \mathcal{E}_{\pi(p)}=f(p)$ and so $G \in \mathcal{F}$, a contradiction.

Therefore $G$ is a primitive group of type 2 and, in particular, $2 \in \pi(N)$. Note that any normal subgroup of $G$, in particular $N$, belongs to $\mathcal{L}$.

It is known that no simple group $E$ has nilpotent Hall $\nu$-subgroups with $\nu$ a set of primes such that $|\nu \cap \pi(E)|>1$ and $2 \in \nu$ (see [1, Proposition 1]). Hence any non-abelian simple group in $\mathcal{L}$ belongs to $\mathcal{E}_{\pi(2)}$; consequently, $N \in \mathcal{E}_{\pi(2)} \subseteq \mathcal{F}$ and $N<G$.

On the other hand, the choice of $G$ implies that $M \in \mathcal{F} \subseteq \mathcal{E}_{2^{\prime}} \mathcal{E}_{\pi(2)}$ for a maximal normal subgroup $M$ of $G$. Consequently $M \in \mathcal{E}_{\pi(2)}$ because $N \leq M$ and then $O_{2^{\prime}}(M)=1$.

Since $G \notin \mathcal{E}_{\pi(2)}$, there is a prime $p \in \pi(G) \backslash \pi(2)$ and $|G / M|=p$ as $G / M \in \mathcal{F}=\mathcal{H} \times \mathcal{E}_{\tau}$. Since $G \in \mathcal{L}$ it follows that $G$ has a normal Sylow p-subgroup $G_{p}$ and $G=M \times G_{p} \in \mathcal{F}$, the final contradiction.

Example 3. Let $\mathcal{F}$ be a covering-formation such that if $2, p \in \pi$ and $p \in$ $\{3,5\}$, then $p$ is not connected with 2. (Equivalently, $\pi=\rho \cup \tau$ with $\rho \cap \tau=\emptyset$, $2 \notin \rho$ and $3,5 \notin \tau)$. Then $\mathcal{F} \subseteq \mathcal{S}$ is a covering-formation of soluble groups. More precisely,

$$
\mathcal{F}=\mathcal{H} \times \mathcal{J}:=\left(G \in \mathcal{E}_{\pi} \mid G=H \times J, H \in \mathcal{H}, J \in \mathcal{J}\right)
$$

where $\mathcal{H}$ and $\mathcal{J}$ are covering-formations of characteristics $\tau$ and $\rho$, respectively. Moreover, $\mathcal{F}=\mathcal{H} \times \mathcal{J}=\bigcap_{\sigma \in \Sigma_{\mathcal{F}}} \mathbf{E}_{\sigma}^{\mathrm{n}}$.

(For instance the following classes of groups appear as particular types of this construction:

$$
\begin{gathered}
\mathcal{H} \times \mathcal{E}_{\{3,5\}}:=\left(G \in \mathcal{E}_{\pi} \mid G=A \times B, A \in \mathcal{H}, B \in \mathcal{E}_{\{3,5\}}\right), \text { or also } \\
\mathcal{H} \times \mathcal{E}_{3} \times \mathcal{E}_{5}:=\left(G \in \mathcal{E}_{\pi} \mid G=A \times B \times C, A \in \mathcal{H}, B \in \mathcal{E}_{3}, C \in \mathcal{E}_{5}\right),
\end{gathered}
$$

where (in both cases) $3,5 \in \pi$ and $\mathcal{H}$ is a covering-formation of soluble groups of characteristic $\pi \backslash\{3,5\}$.)

Proof. The restrictions on the sets of primes imply that $f(p)=\mathcal{E}_{\pi(p)} \subseteq \mathcal{S}$ for any $p \in \pi$. Hence $\mathcal{F} \subseteq \mathcal{S}$. See Lemma 5.3 for the description of $\mathcal{F}$.

On the other hand, by Proposition 5.1 we have $\mathcal{F}=\mathcal{L} \cap \mathcal{S}$ for $\mathcal{L}:=$ $\bigcap_{\sigma \in \Sigma_{\mathcal{F}}} \mathbf{E}_{\sigma}^{\mathrm{n}}$. Let us consider the covering-formation $\mathcal{C}:=\mathcal{E}_{\tau} \times \mathcal{J}$, which is of type given in Example 2. Then $\mathcal{C}=\bigcap_{\sigma \in \Sigma_{\mathcal{C}}} \mathbf{E}_{\sigma}^{\mathrm{n}}$. Note that $\Sigma_{\mathcal{C}} \subseteq \Sigma_{\mathcal{F}}$, which implies that $\mathcal{L}=\bigcap_{\sigma \in \Sigma_{\mathcal{F}}} \mathbf{E}_{\sigma}^{\mathrm{n}} \subseteq \bigcap_{\sigma \in \Sigma_{\mathcal{C}}} \mathbf{E}_{\sigma}^{\mathrm{n}}=\mathcal{C} \subseteq \mathcal{S}$. Consequently $\mathcal{F}=\mathcal{L}$ and we are done. 
Remark 5.4. (1) For covering-formations, in the universe of all finite groups, we know that $\mathcal{F} \subseteq \mathrm{N} \mathcal{F} \subseteq \mathcal{E}_{\pi}$ since $\mathcal{F}$ is subgroup-closed, but in general $\mathcal{F} \neq \mathrm{N} \mathcal{F}$. (See [12, Remark 1(c)].)

(2) A subgroup-closed saturated formation $\mathcal{X}$ with $\mathrm{N} \mathcal{X}=\mathcal{X}$ need not be a covering-formation. (See [10].)

(3) As pointed out also in [12], it follows from the Feit-Thompson theorem and Theorem 5.2 that if $2 \notin \pi$, then $\mathcal{F}=\mathrm{N} \mathcal{F}$. The result $\mathcal{F}=\mathrm{N} \mathcal{F}$ can be deduced in the same way under the hypothesis that $3,5 \notin \pi$.

Proposition 5.5. Let $G$ be a group of minimal order in $\mathrm{N} \mathcal{F} \backslash \mathcal{F}$. Then $G$ is a primitive group of type 2 and any pair of primes in $\pi(G)$ is connected.

Proof. Note that $\mathrm{N} \mathcal{F}$ is Q-closed because $N_{G / N}\left(G_{p} N / N\right)=N_{G}\left(G_{p}\right) N / N$ for any $G_{p} \in \operatorname{Syl}_{p}(G)$ and $N \unlhd G$. Since $\mathcal{F}$ is a saturated formation we can deduce that $G$ is a primitive group of type either 1 or 2, i.e., with a unique minimal normal subgroup, say $N$.

Assume that $G$ is primitive of type 1 . Then $N=C_{G}(N)$ is a $p$-group, for some prime $p$, and $G=N M$ with $M$ a maximal subgroup of $G, N \cap M=1$.

We claim that $G / N \in \mathcal{E}_{\pi(p)}=f(p)$.

If $p \notin \pi(G / N)$, then $N \in \operatorname{Syl}_{p}(G)$ and $G=N_{G}(N) \in \mathcal{F}$, a contradiction.

Hence $p \in \pi(G / N)$. Assume that there exists $q \in \pi(G / N), q \notin \pi(p)$. Observe that $M \cong G / N \in \mathcal{F} \subseteq \mathcal{E}_{p^{\prime}} \mathcal{E}_{\pi(p)}$. In particular we can consider $1 \neq M_{q} \in \operatorname{Syl}_{q}(M)$ and $M_{q} \leq O_{p^{\prime}}(M)$. By Lemma 2.1 it follows that $M=O_{p^{\prime}}(M) N_{M}\left(M_{q}\right)$. Then there exists $1 \neq M_{p} \in \operatorname{Syl}_{p}(M)$ with $M_{p} \leq$ $N_{M}\left(M_{q}\right) \in \mathcal{F}$. Since $p \notin \pi(q)$ it follows that $\left[M_{p}, M_{q}\right]=1$. Consequently $M_{q} \leq N_{G}\left(N M_{p}\right) \in \mathcal{F}$ as $N M_{p} \in \operatorname{Syl}_{p}(G)$ and $G \in \mathrm{N} \mathcal{F}$. Again since $q \notin \pi(p)$ it follows that $M_{q} \leq C_{G}(N)=N$, a contradiction which proves the claim.

Since $G / N \in \mathcal{F}$ and $G / N=G / C_{G}(N) \in \mathcal{E}_{\pi(p)}=f(p)$ it follows that $G \in \mathcal{F}$, a contradiction.

Therefore $G$ is a primitive group of type 2 . Hence $N=N_{1} \times \ldots \times N_{r}$, where $N_{i} \cong N_{j}$ are non-abelian simple groups for any $i, j \in\{1, \ldots, r\}$. Set $R=\cap_{i=1}^{r} N_{G}\left(N_{i}\right) \unlhd G$.

Assume first that $R<G$. We claim that if $p \in \pi(N)$ and $q \in \pi(G / R)$, then $p \leftrightarrow q$. This will imply the result.

Since $G / R \in \mathcal{F}$ we have, in particular, that $G / R \in \mathcal{E}_{q^{\prime}} \mathcal{E}_{\pi(q)}$. If $p \notin \pi(q)$ there exists $G_{p} \in \operatorname{Syl}_{p}(G)$ such that $G_{p} \leq T \unlhd G$ with $T / R=O_{q^{\prime}}(G / R)$. By Lemma 2.1, $G=T N_{G}\left(G_{p}\right)$ and there exists a subgroup $T_{q} Q \in \operatorname{Syl}_{q}(G)$ for some $T_{q} \in \operatorname{Syl}_{q}(T)$ and some $Q \in \operatorname{Syl}_{q}\left(N_{G}\left(G_{p}\right)\right)$. Since $N_{G}\left(G_{p}\right) \in \mathcal{F}$ and $q \notin \pi(p)$ it follows that $\left[G_{p}, Q\right]=1$ and thus $\left[N_{p}, Q\right]=1$, where $1 \neq N_{p}=$ $G_{p} \cap N \in \operatorname{Syl}_{p}(N)$. But $R<G$. Hence $r>1$ and $N_{p}=\left(N_{1}\right)_{p} \times \ldots \times\left(N_{r}\right)_{p}$ 
with $\left(N_{i}\right)_{p} \in \operatorname{Syl}_{p}\left(N_{i}\right)$ for every $i=1, \ldots, r$. Since $G$ acts transitively via conjugation on the set $\left\{N_{1}, \ldots, N_{r}\right\}$, we deduce that $Q \leq R$ and therefore $T_{q} Q \leq R$, a contradiction.

Assume now that $G=R$. Then $r=1$ and $G$ is an almost simple group. We claim that $\pi\left(A_{p}(G)\right) \subseteq \pi(p)$ for every prime $p \in \pi(G)$. Since $N_{G}\left(G_{p}\right) \in \mathcal{F}$ for $G_{p} \in \operatorname{Syl}_{p}(G)$, if $q \in \pi\left(N_{G}\left(G_{p}\right)\right)$ and $q \notin \pi(p)$ we have that $\left[Q, G_{p}\right]=1$ for any $Q \in \operatorname{Syl}_{q}\left(N_{G}\left(G_{p}\right)\right)$. Therefore $q \notin \pi\left(A_{p}(G)\right)$ and the claim is proved. The result can be deduced now from the Main Theorem.

This proposition can be useful to derive conditions assuring that $\mathrm{N} \mathcal{F}=$ $\mathcal{F}$. In particular we deduce next that for any of the classes of groups in Examples 1, 2 and 3, a group belongs to the class if and only if its Sylow normalizers belong to the class.

Corollary 5.6. For any of the classes of groups in Example 1, a group belongs to the class if and only if its Sylow normalizers belong to the class.

Proof. Let $\mathcal{F}$ be a covering-formation as in Example 1. Since $\mathcal{F}$ is subgroupclosed, we need only to prove that $\mathrm{N} \mathcal{F} \subseteq \mathcal{F}$. Assume that this is wrong and let $G$ be a group of minimal order in $\mathrm{N} \mathcal{F} \backslash \mathcal{F}$. Then Proposition 5.5 implies that $G$ is a primitive group of type 2 and each pair of primes $p, q \in \pi(G)$ is connected. In particular, every prime $p \in \pi(G) \backslash\{2\}$ is connected with $2 \in$ $\pi(G)$ and consequently $\pi(p)=\pi(2)$ and $G \in \mathcal{E}_{\pi(2)} \subseteq \mathcal{F}$, a contradiction.

Remark. Observe that the proof of Corollary 5.6 can be adapted to derive the corresponding results for the covering-formations in Examples 2 and 3. We show below a more direct approach to these results based on Corollary 5.6.

Lemma 5.7. Let $\left\{\tau_{i} \mid i \in I\right\}$ be a partition of a set of primes $\tau$ and let $\mathcal{X}$ and $1 \in \mathcal{X}_{i} \subseteq \mathcal{E}_{\tau_{i}}$ for every $i \in I$ be classes of groups such that

$$
\mathcal{X}:=\times_{i \in I} \mathcal{X}_{i}:=\left(G \in \mathcal{E}_{\tau} \mid G=\times_{i \in I} G_{\tau_{i}}, G_{\tau_{i}} \in \mathcal{X}_{i}\right) .
$$

Then $\mathrm{N} \mathcal{X}=\mathcal{X}$ if and only if $\mathrm{N} \mathcal{X}_{i}=\mathcal{X}_{i}$ for all $i \in I$.

Proof. This follows by an straightforward proof and Corollary 5.6.

Corollary 5.8. For any of the classes of groups in Examples 2 and 3, a group belongs to the class if and only if its Sylow normalizers belong to the class.

Proof. It follows by Lemma 5.7 and Remark 5.4 (3). 
Remark. As pointed out in Remark 5.4 (2), there is an example in [10] of a subgroup-closed saturated formation $\mathcal{X}$ with the property n $\mathcal{X}=\mathcal{X}$ which is not a covering-formation. This example depends on the primes in the characteristic of $\mathcal{X}$ and on the non-abelian simple groups involving these primes as divisors of their orders. Here $\sigma:=\operatorname{Char}(\mathcal{X})=\{2,5,7,13, p\}$, where $p \neq 2,3,5,7,13$, and it is proved that the Suzuki group $\mathrm{Sz}\left(2^{3}\right)$ is the only non-abelian simple $\sigma$-group.

We may wonder whether there is an example of such a formation with full characteristic. Lemma 5.7 provides an easy way to construct an example as aimed from the saturated formation $\mathcal{X}$ in [10]. We need just to consider now the subgroup-closed saturated formation $\mathcal{H}:=\mathcal{X} \times \mathcal{E}_{\sigma^{\prime}}$ with $\sigma=\operatorname{Char}(\mathcal{X})$.

\section{References}

[1] Z. Arad, D. Chillag, Finite groups containing a nilpotent Hall subgroup of even order, Houston J. Math. 7 (1) (1981), 23-32.

[2] H. Azad, Semi-simple elements of order 3 in finite Chevalley groups, J. Algebra 56 (1979), 481-498.

[3] A. Ballester-Bolinches, A. Martínez-Pastor, M. C. Pedraza-Aguilera, M. D. Pérez-Ramos, On nilpotent-like Fitting formations, in: C. M. Campbell et al.(Eds.), Proceedings of Groups-St Andrews 2001 in Oxford, Lond. Math. Soc. Lect. Note Ser. 304, Vol. 1, Cambridge University Press, 2003, pp. 31-38.

[4] M. G. Bianchi, A. Gillio Berta Mauri, P. Hauck, On finite groups with nilpotent Sylow normalizers, Arch. Math. 47 (1986), 193-197.

[5] A. Borel, R. Carter, C. W. Curtis, N. Iwahori, T. A. Springer, R. Steinberg, Seminar on algebraic groups and related finite groups, Lecture Notes Math. 131, Springer, Berlin, 1970.

[6] N. Bourbaki, Éléments de mathématique: Groupes et algèbres de Lie, Chapters IV, V, VI, Hermann, Paris, 1968.

[7] R. W. Carter, Simple groups of Lie type, Wiley, London, 1972.

[8] R. W. Carter, Finite groups of Lie type: Conjugacy classes and complex characters, Wiley, London, 1985.

[9] R. W. Carter, Conjugacy classes in the Weyl group, Compos. Math. 25 (1972), 1-59. 
[10] A. D'Aniello, C. De Vivo, G. Giordano, On certain saturated formations of finite groups, in: T. Hawkes, P. Longobardy, M. Maj (Eds.) Proceedings Ischia Group Theory 2006, World Scientific, Hackensack, NJ, 2007, pp. 22-32.

[11] A. D'Aniello, C. De Vivo, G. Giordano, Lattice formations and Sylow normalizers: a conjecture, Atti Semin. Mat. Fis. Univ. Modena Reggio Emilia 55 (2007), 107-112.

[12] A. D'Aniello, C. De Vivo, G. Giordano, M. D. Pérez-Ramos, Saturated formations closed under Sylow normalizers, Comm. Algebra 33 (2005), 2801-2808.

[13] K. Doerk, T. Hawkes, Finite soluble groups, Walter De Gruyter, Berlin-New York, 1992.

[14] G. Glauberman, Prime-power factor groups of finite groups II, Math. Z. 117 (1970), 46-56.

[15] D. Gorenstein, R. Lyons, The local 2-structure of groups of characteristic 2 type, Mem. Am. Math. Soc., vol. 42, N. 276, Providence, R.I., USA, 1983.

[16] R. M. Guralnick, G. Malle, G. Navarro, Self-normalizing Sylow subgroups, Proc. Amer. Math. Soc. 132 (2004), 973-979.

[17] F. Menegazzo, M. C. Tamburini, A property of Sylow p-normalizers in simple groups, Quaderni del seminario Matematico di Brescia, n.45/02 (2002).

[18] R. Steinberg, Lectures on Chevalley groups, Yale Univ., 1967.

[19] E. Stensholt, An application of Steinberg's construction of twisted groups, Pac. J. Math. 55 (1974), 595-618.

[20] E. Stensholt, Certain embeddings among finite groups of Lie type, J. Algebra 53 (1978), 136-187.

[21] K. Zsigmondy, Zur Theorie der Potenzreste, Monatsh. Math. Phys. 3 (1892), 265-284. 